\title{
Epidural tramadol via intraoperatively placed catheter as a standalone analgesic after spinal fusion procedure: An analysis of efficacy and cost
}

\author{
Vijaysundar Ilangovan, Thanga Tirupathi Rajan Vivakaran, D. Gunasekaran ${ }^{1}$, D. Devikala ${ }^{1}$ \\ Departments of Neurosurgery and ${ }^{1}$ Anaesthesiology, Saveetha Medical College and Hospital, Kanchipuram, Tamil Nadu, India
}

\begin{abstract}
Objective: This was a prospective analysis of epidural tramadol as a single analgesic agent delivered through intraoperatively placed epidural catheter for postoperative pain relief after spinal fusion procedures in terms of efficacy and cost. Materials and Methods: Twenty patients who underwent spinal fusion procedures were included in the study. After completion of the procedure, an epidural catheter was placed at the highest level of exposed dura and brought out through a separate tract. Postoperatively, tramadol was infused into the epidural space via the catheter at a dose of $1 \mathrm{mg} / \mathrm{kg}$ diluted in $10 \mathrm{ml}$ of saline. The dosage frequency was arbitrarily fixed at every $6 \mathrm{~h}$ during the first 2 days and thereafter reduced to every $8 \mathrm{~h}$ after the first 2 days till day 5. Conventional intravenous analgesics were used only if additional analgesia was required as assessed by visual analog scale (VAS). Results: Patients' VAS score was assessed every $4 \mathrm{~h}$ from the day of surgery. Patients with a VAS score of 6 or more were given additional analgesia in the form of intravenous paracetamol. Of the twenty patients, eight patients needed additional analgesia during the first $24 \mathrm{~h}$ and none required additional analgesia after the first $24 \mathrm{~h}$. The median VAS score was 7 within the first $24 \mathrm{~h}$ and progressively declined thereafter. Epidural tramadol was noted to be many times cheaper than conventional parenteral analgesics. Conclusion: Epidural tramadol infusion is safe and effective as a standalone analgesic after open spinal fusion surgery, especially after the $1^{\text {st }}$ postoperative day. Intraoperative placement of the epidural catheter is a simple way of delivering tramadol to the epidural space. The cost of analgesia after spinal fusion surgery can be reduced significantly using epidural tramadol alone.
\end{abstract}

Key words: Cost-effectiveness, epidural analgesia, spinal fusion, tramadol

\section{Introduction}

Traditional posterior lumbar interbody fusion and posterior transpedicular screw fixation are associated with high degree of postoperative pain. Most patients require parenteral administration of analgesics or epidural opioids, especially during the first 2 days after operations or more. We chose to use tramadol

\section{Address for correspondence:}

Dr. Vijaysundar Ilangovan, No. 6 Bharathi Nagar, $1^{\text {st }}$ Street,

North Usman Road, T. Nagar, Chennai - 600 017, Tamil Nadu, India. E-mail: drvijaysundar@gmail.com

\begin{tabular}{|l|l|}
\hline \multicolumn{2}{|c|}{ Access this article online } \\
\hline Quick Response Code: & Website: \\
\hline & www.ruralneuropractice.com \\
\cline { 2 - 2 } & \\
\hline & \\
\hline
\end{tabular}

alone as postoperative analgesia delivered through an intraoperatively placed epidural catheter to see if the costs of conventional parenteral analgesics and the side effects of epidural opioids can be avoided while maintaining the benefit of epidural analgesia since high degree of postoperative pain precludes early mobilization, which in turn lengthens hospital stay and might result in various complications. ${ }^{[1]}$ We undertook an analysis of twenty patients who underwent spinal fusion procedure and were given postoperative epidural tramadol alone and analyzed the results in terms of efficacy and cost.

This is an open access article distributed under the terms of the Creative Commons Attribution-NonCommercial-ShareAlike 3.0 License, which allows others to remix, tweak, and build upon the work non-commercially, as long as the author is credited and the new creations are licensed under the identical terms.

For reprints contact: reprints@medknow.com

How to cite this article: llangovan V, Vivakaran TT, Gunasekaran D, Devikala D. Epidural tramadol via intraoperatively placed catheter as a standalone analgesic after spinal fusion procedure: An analysis of efficacy and cost. J Neurosci Rural Pract 2017;8:55-9. 


\section{Materials and Methods}

We included twenty consecutive patients who underwent lumbar spinal fusion surgery for degenerative disc disease, spondylolisthesis, and spinal instability between December 2014 and January 2016. Exclusion criteria were American Society of Anesthesiologists (ASA) Grade 3 or more, intraoperative dural rent, or patients with severe comorbidities. Written informed consent was obtained from all patients. There were 12 male and 8 female patients in our study, with a male to female ratio of 3:2. The mean age of the patients was 57.65 years and the median weight was $71 \mathrm{~kg}$. All patients were ASA Grade 1 or 2 , with a ratio of 11:9. The median duration of surgery was $3.36 \mathrm{~h}$ [Table 1].

All patients were managed by the same team (anesthesiologist/neurosurgeons) and intraoperative fentanyl was used. All patients underwent posterolateral spinal fusion procedure as usual, and at the end of the procedure, an epidural catheter was placed at the highest level of exposed dura. In case the dura was not exposed, a small fenestration was made at the highest interlaminar space that was exposed and the epidural catheter was inserted through it. The epidural catheter was passed retrograde through the needle in the paraspinal muscles after removal of the stylet by the surgeon before wound closure. No additional exposure or increase of incision was used to accommodate the epidural catheter.
All patients were given tramadol via epidural catheter at a dose of $1 \mathrm{mg} / \mathrm{kg}$ diluted in $10 \mathrm{ml}$ of normal saline with the first dose starting immediately after closure. Epidural tramadol was given 4 times a day at a dosage of $1 \mathrm{mg} / \mathrm{kg}$ for a minimum of 2 days after which the dosage was reduced to 3 times a day till day 5 . Patient was then prescribed conventional oral analgesics and epidural catheter was removed. Postoperatively, pain was assessed with a visual analog scale (VAS) using a $10 \mathrm{~cm}$ plastic scale ranging between 0 (no pain) and 10 (worst imaginable pain) [Figure 1]. Patients were instructed to define their pain by the scale every $4 \mathrm{~h}$ during the first postoperative $24 \mathrm{~h}$. Patients who had VAS score of 6 or more were given additional intravenous analgesia in the form of paracetamol infusions of $1 \mathrm{~g}(100 \mathrm{ml})$ stat only. The side effect profile in the first postoperative $24 \mathrm{~h}$ was also recorded. The removal of the wound site drain and sutures were as per the usual norms.

\section{Results}

All of the patients were able to complete the study. There was no significant difference between patients in terms of age, weight, height, sex, ASA, physical status, and durations of operation and anesthesia. All surgeries were performed by the same team and on the lumbar spine alone. All patients underwent pain assessment every $4 \mathrm{~h}$ using the conventional VAS [Figure 1]. Patients were administered additional parenteral analgesia if the VAS

Table 1: Master chart

\begin{tabular}{|c|c|c|c|c|c|}
\hline Age & Sex & Weight & Height & ASA & Duration (h) \\
\hline 48 & Female & 58 & 156 & 1 & 4 \\
\hline 58 & Female & 68 & 158 & 2 & 3.5 \\
\hline 64 & Male & 72 & 160 & 2 & 3.2 \\
\hline 55 & Male & 82 & 178 & 1 & 4.1 \\
\hline 52 & Female & 65 & 162 & 2 & 3.8 \\
\hline 65 & Male & 68 & 180 & 1 & 4 \\
\hline 64 & Male & 70 & 164 & 1 & 2.8 \\
\hline 59 & Male & 80 & 178 & 2 & 3 \\
\hline 63 & Female & 62 & 154 & 1 & 4.5 \\
\hline 61 & Male & 84 & 162 & 2 & 3.6 \\
\hline 59 & Male & 76 & 180 & 1 & 2.7 \\
\hline 57 & Male & 69 & 168 & 1 & 3.5 \\
\hline 63 & Female & 68 & 160 & 1 & 3 \\
\hline 58 & Male & 78 & 174 & 2 & 4.2 \\
\hline 54 & Male & 84 & 182 & 2 & 3.5 \\
\hline 49 & Female & 70 & 165 & 1 & 3.9 \\
\hline 53 & Male & 80 & 176 & 2 & 2.9 \\
\hline 63 & Female & 64 & 170 & 1 & 4 \\
\hline 59 & Male & 68 & 168 & 2 & 3 \\
\hline 49 & Female & 55 & 153 & 1 & 3.7 \\
\hline 57.65 & Male: female $=3: 2$ & 71.05 & 167.4 & ASA $1:$ ASA $2=11: 9$ & 3.36 \\
\hline
\end{tabular}




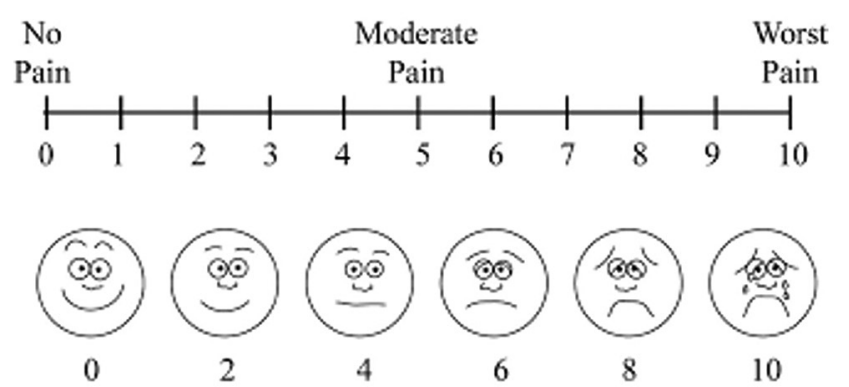

Figure 1: Visual analog scale

score was 6 or more. Of the twenty patients, eight patients needed additional analgesia during the first $24 \mathrm{~h}$ and none required additional analgesia after the first $24 \mathrm{~h}$. The median VAS score was 7 within the first $24 \mathrm{~h}$ and progressively declined thereafter [Figure 2].

The number of dosages of tramadol ranged from 17 to 19 for the first 5 days (1-3 on postoperative day [POD] 0,8 on POD 1 and POD 2, and 9 for the next 3 PODs). The cost of analgesics alone for the first 5 PODs ranges from 442 to 494 Indian national rupees (INR), with an additional cost of 980 INR for the epidural catheter.

The incidence of side effects was very low with just two of the twenty patients reporting nausea or vomiting which settled spontaneously. All the four patients who required additional analgesia during the first $24 \mathrm{~h}$ required it only once. There were no complications related to the epidural catheter such as infection or avulsion. All of the patients also had a drain inserted opposite to the side that the catheter was inserted. Drain removal was as per the usual norms.

\section{Discussion}

Multilevel spinal surgery is becoming increasingly common even in elderly patients. ${ }^{[2]}$ This involves consumption of large amounts of nonsteroidal anti-inflammatory drugs and opioids which hinder postoperative mobilization and rehabilitation ${ }^{[3]}$ Although there are many numbers of studies that have focused on pain treatment in spine surgery, they are mostly focused on conventional analgesia and opioids. Different pain mechanisms such as neuropathic inflammatory and nociceptive contribute to postoperative pain in addition to peripheral and central sensitization. Epidural analgesia has been used successfully for postoperative analgesia after spinal fusion procedures, but most of the studies have used epidural opioids or used multiple drugs, both of which require the patient to be monitored, which in turn spikes up costs involved and may cause additional side effects.

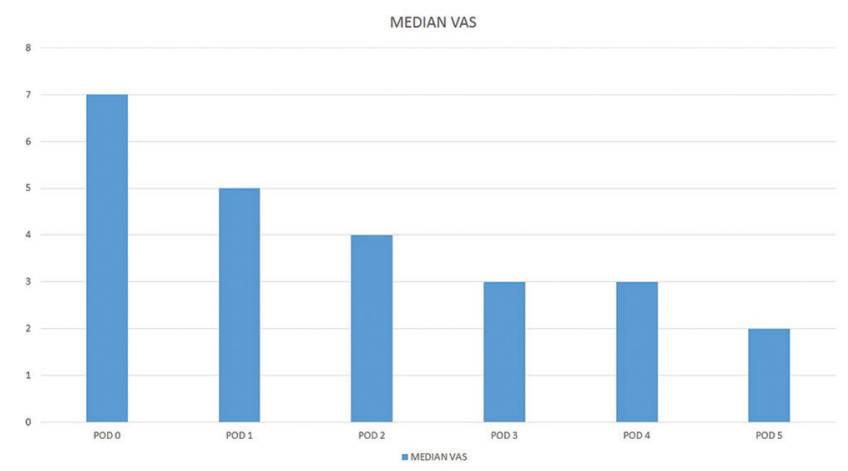

Figure 2: Median visual analog scale

Tramadol hydrochloride is a synthetic codeine analog that has opioid and nonopioid properties. ${ }^{[4]}$ It decreases pain in the spinal cord with a weak affinity to the $\mu$-opioid receptors by inhibiting noradrenaline and serotonin reuptake. Tramadol and its metabolites are mainly excreted via the kidneys. The mean elimination half-life is about $6 \mathrm{~h}$. The analgesic potency of tramadol is about $10 \%$ of that of morphine, following parenteral administration. ${ }^{[5]}$ Tramadol provides postoperative pain relief comparable with that of pethidine. Tramadol is an effective and well-tolerated agent to reduce pain resulting from trauma, renal or biliary colic, and labor, and also for the management of chronic pain of malignant or nonmalignant origin, particularly neuropathic pain. Although tramadol is thought to act via a different mechanism for alleviating inflammatory pain, it decreased the concentration of proinflammatory cytokines in the spinal cord of rats. ${ }^{[6]}$ The side effect profile is milder when compared with the strong opioids, and tramadol via the epidural route has been proven safe according to large-scale studies. The benefits of using tramadol as a standalone analgesic in postoperative pain relief in spinal fusion include safety, better side effect profile, low cost, and the fact that analgesia can be administered in the general ward. A reduction in opioid consumption and related side effects is also a benefit. ${ }^{[7]}$ The safety of epidural tramadol has previously been demonstrated, and the epidural route for delivering postoperative analgesia after spinal fusion procedures is well validated. ${ }^{[3]}$

In our study, all patients underwent spinal fusion procedure as usual, and on completion of the procedure, an epidural catheter was inserted through a separate tract and the tip placed at the level of the highest exposed dura. No additional exposure was made use of. Epidural infusions of tramadol were started immediately after skin closure and continued for every $6 \mathrm{~h}$ for the first 2 days. A wound site drain was placed as usual. Of the twenty patients in our study, eight patients required additional 
analgesics in the form of intravenous paracetamol during the first $24 \mathrm{~h}$. The median VAS score was 7 during the day of surgery but progressively declined to 5, 4, and 3 over the next 3 days. All twenty patients had a wound site drain inserted in the operative field, and this did not hamper the analgesic effect of the drug. Except for the $1^{\text {st }}$ POD, all patients were managed in the general wards and epidural tramadol was administered by the ward nurse under doctor's supervision.

In an institute such as ours where the majority of the patients are poor and can only undergo surgeries involving implants under government health insurance schemes, cost is an important factor to consider. In that aspect, each vial of tramadol costs 26 INR and contains $100 \mathrm{mg}$ in $2 \mathrm{ml}$ whereas paracetamol infusions are available in $100 \mathrm{ml}$ bottles containing $1 \mathrm{~g}$ of paracetamol which costs 302 INR. The per day cost of analgesia using tramadol is more than 10 times lesser than that of using conventional analgesia. The epidural catheter costs around 980 INR. Even if the added cost of using additional analgesics for the $1^{\text {st }}$ day and the cost of the epidural catheter are considered, tramadol is still far more cost-effective [Figure 3]. Although some previous studies have opined that epidural patient-controlled analgesia is costlier, those studies have compared it against intrathecal morphine, have used multiple drugs, and hence have to factor in the extra cost of equipment required to monitor patients receiving multiple epidural drugs. ${ }^{[8]}$

The side effect profile of tramadol via the epidural route is quite low as compared to other opioids delivered via the same route which can cause side effects such as nausea and vomiting or more grievous one like ileus, respiratory depression, and motor block. ${ }^{[9]}$ An additional advantage of tramadol is easy and widespread availability without need of specific drug license unlike morphine or other opioids. Tramadol also has the benefit of none or very low incidence of grave side effects such as respiratory depression which permits administration in the ward.

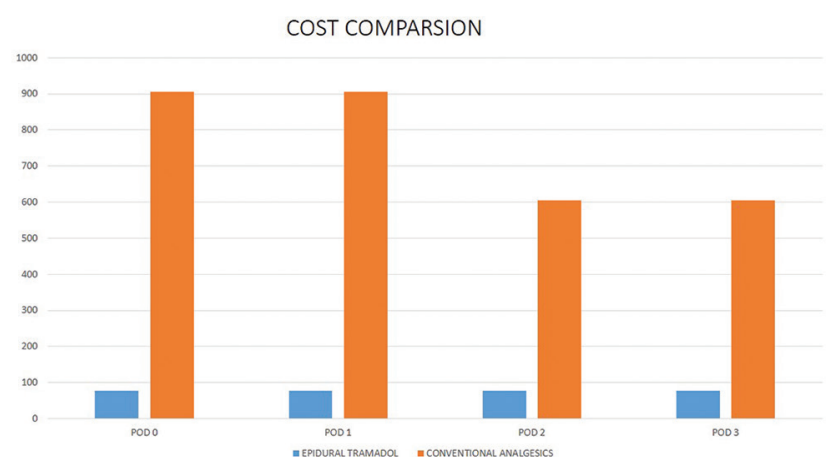

Figure 3: Cost comparison of epidural tramadol and conventional parenteral analgesics
Since we restricted our study to surgeries on the lumbar spine and used only epidural tramadol, we were able to keep our patients in the general ward after the $1^{\text {st }} P O D$, where the ward nurse was instructed to give the epidural tramadol. This helped us cut the costs further and avoid intensive care unit/high dependency unit (ICU/HDU) stay as much as possible. We opine that this feature will enable smaller hospitals with limited ICU/HDU capacity to adopt this method of postoperative pain relief.

Some of the limitations of our study were that it was not a comparative analysis of epidural tramadol against conventional analgesics, all of the patients in the study had surgeries done on their lumbar spine, and that the sample size was not too big. On the other hand, some interpretations we can make are that an intraoperative epidural catheter can be placed during surgery and used for postoperative analgesia without affecting the postoperative recovery in any way. Even the presence of wound drain does not reduce the efficacy of epidural tramadol. Tramadol via the epidural catheter can be administered in the general ward without the need for any special monitoring equipment.

\section{Conclusion}

Pain relief after major spinal fusion surgery can be effectively administered via epidural catheter. Tramadol is a safe and effective drug for the purpose of epidural analgesia with a low side effect profile that reduces the need for patient monitoring and enables administration of epidural tramadol in the general ward. The cost of postoperative analgesia for the first 5 days using epidural tramadol alone is many times lower than conventional intravenous analgesics. Further studies comparing tramadol alone as an epidural analgesic against conventional epidural analgesics involving higher doses of tramadol may provide further information.

\section{Acknowledgment}

We acknowledge that all authors have contributed equally to the preparation of the manuscript.

\section{Financial support and sponsorship \\ Nil.}

\section{Conflicts of interest}

There are no conflicts of interest.

\section{References}

1. Lee SH, Kim KH, Cheong SM, Kim S, Kooh M, Chin DK. A comparison of the effect of epidural patient-controlled analgesia with intravenous 
patient-controlled analgesia on pain control after posterior lumbar instrumented fusion. J Korean Neurosurg Soc 2011;50:205-8.

2. Pumberger M, Chiu YL, Ma Y, Girardi FP, Mazumdar M, Memtsoudis SG. National in-hospital morbidity and mortality trends after lumbar fusion surgery between 1998 and 2008. J Bone Joint Surg Br 2012;94:359-64.

3. Gottschalk A, Freitag M, Tank S, Burmeister MA, Kreil S, Kothe R, et al. Quality of postoperative pain using an intraoperatively placed epidural catheter after major lumbar spinal surgery. Anesthesiology 2004;101:175-80.

4. Şahin Y, Apan A, Öz G, Evliyaoğlu CA. Epidural tramadol infiltration decreases postoperative analgesic consumption after lumbar microdiscectomy. Turk J Med Sci 2012;42:395-401.
5. Grond S, Sablotzki A. Clinical pharmacology of tramadol. Clin Pharmacokinet 2004;43:879-923.

6. Carlsson KH, Jurna I. Effects of tramadol on motor and sensory responses of the spinal nociceptive system in the rat. Eur J Pharmacol 1987;139:1-10.

7. White PF, Kehlet H. Improving postoperative pain management: What are the unresolved issues? Anesthesiology 2010;112:220-5.

8. Vercauteren M, Vereecken K, La Malfa M, Coppejans H, Adriaensen H. Cost-effectiveness of analgesia after caesarean section. A comparison of intrathecal morphine and epidural PCA. Acta Anaesthesiol Scand 2002;46:85-9.

9. Schenk MR, Putzier M, Kügler B, Tohtz S, Voigt K, Schink T, et al. Postoperative analgesia after major spine surgery: Patient-controlled epidural analgesia versus patient-controlled intravenous analgesia. Anesth Analg 2006;103:1311-7. 